\title{
Improving Efficiency, Linearity and Linearisability of an Asymmetric Doherty Power Amplifier by Modulating the Peaking Amplifier's Supply Voltage
}

\author{
Alexander Alt, Jonathan Lees \\ School of Engineering, Cardiff University, Cardiff, United Kingdom
}

\begin{abstract}
Envelope tracking and asymmetric Doherty power amplifiers are two techniques that can be used to achieve high average efficiencies when amplifying signals with high peak-toaverage power ratios, e.g. those employed in modern wireless communication standards. In this paper, a combination of the two techniques, the modulation of the drain voltage of the peaking amplifier in an asymmetric Doherty power amplifier, is described. It is demonstrated how this combination can increase the efficiency in the high-power range while reducing the AM/PM distortion of the DPA. Additionally, the degrees of freedom in terms of efficiency, linearity, and linearisability when designing the shaping function is discussed. Measurements of an asymmetric $2.4 \mathrm{GHz}$ DPA using $10 \mathrm{~W}$ and $25 \mathrm{~W}$ GaN HEMTs prove the concept and demonstrate the achievable improvements in terms of efficiency, linearity, and linearisability.

Index Terms-Doherty power amplifiers, envelope tracking, power amplifiers, pre-distortion, wireless communication.
\end{abstract}

\section{INTRODUCTION}

Driven by modern communication standards with high peak to average power ratios (PAPR) and growing bandwidths, power amplifiers need to achieve high efficiencies over a wide power range with good linearity over these wide bandwidths. High efficiency across a wide bandwidth can be achieved using harmonic tuning techniques such as continuous mode amplifiers [1]. To reach high efficiencies over a wide power range, techniques including Doherty power amplifiers (DPA), outphasing structures and envelope tracking (ET) architectures have been established. To achieve reasonable efficiency over the $11 \mathrm{~dB}$ of dynamic range necessary for LTE [2], a DPA needs to be quite asymmetric which results in a deep efficiency dip between the transition point (TP) and peak envelope power (PEP), cf. Fig. $1 b$.

- To achieve a high dynamic range with high efficiency, we propose a combination of envelope tracking and the DPA structure. The benefits of this approach are described in section II.

- In section III, we simulate the behaviour of the amplifier with different peaking device drain voltages and explore the possibilities to identify ways to optimise for linearity, linearisability, and efficiency.

- A DPA is designed and measured with different peaking drain voltages, verifying our assumptions and simulations. In section IV, we compare these and discuss the results.

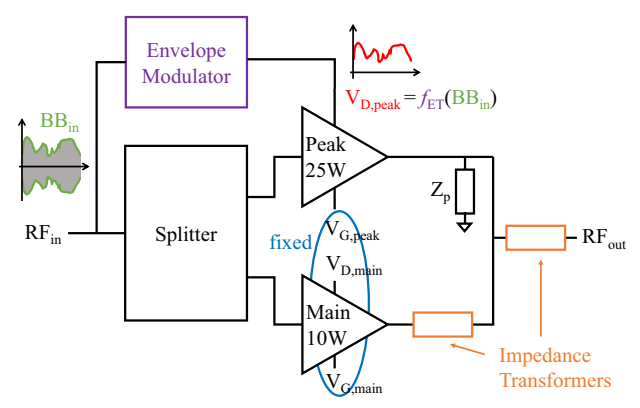

(a)

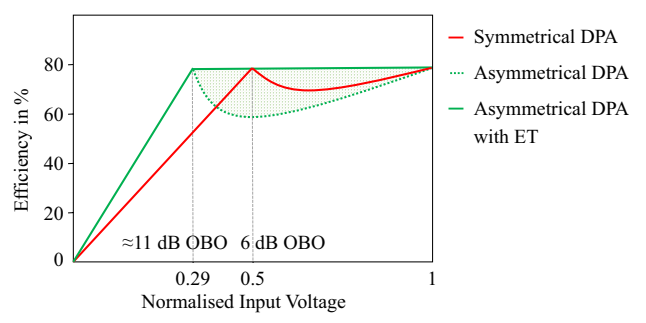

(b)

Fig. 1. Schematic of proposed concept (a), Comparison of the efficiency of symmetrical DPA and asymmetrical DPA with and without ET (ideal class B PAs and ideal modulator) (b)

\section{CONCEPT}

The application of a modulated supply voltage to a DPA has been explored in a number of publications on the combination of ET and DPA, predominately aiming to shape the gate voltages of either peaking or both main and peaking amplifier, [3], the main amplifier's drain voltage [4] or both main and peaking amplifier's drain voltages at the same time [5]. The method of modulating the peaking amplifier's drain voltage proposed in [6] is further developed in this work using supply modulation on an asymmetric DPA, with the aim to achieve $11 \mathrm{~dB}$ of efficient dynamic range, see Fig. 1a. As discussed in [7, p.44], selecting a larger peaking device will provide for an increased high efficiency dynamic range, see Fig. 1b. This dynamic range comes at a cost however; as the peaking amplifier is significantly larger, its impact on the overall efficiency in increased. This leads to a 'deeper' efficiency dip compared to the symmetric DPA, see Fig. 1b. By applying ET to the peaking amplifier as shown in Fig. 1a, the total efficiency of the DPA can be improved, as depicted in Fig. 1 b. 


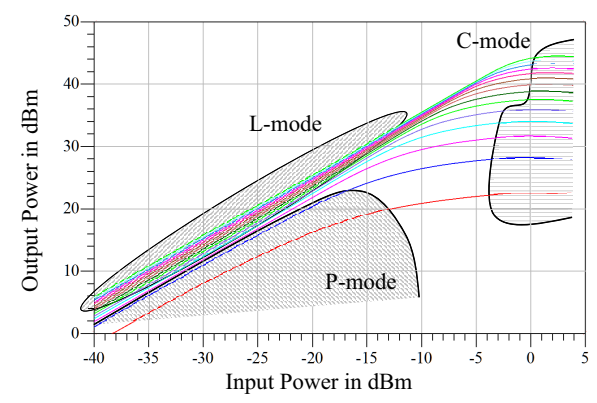

Fig. 2. A booth chart, output power over input power of a simulated $10 \mathrm{~W}$ GaN HEMT PA for different supply voltages

Conventional ET is limited to the L-mode region, where the output power depends predominately on the input power, see Fig. 2 [8]. In this region, a change of drain voltage will increase the efficiency without influencing output power or gain of the amplifier. Real amplifiers will react differently depending on the technology, e.g. the GaN HEMT PA in Fig. 2 shows a slight reduction in gain. Keeping the peaking amplifier in this range will thus increase the efficiency with little influence on the DPA. C-mode operation is typically the domain of EER, the transistor operates in compression, deep in the knee region. In this mode the output power almost exclusively depends on the supply voltage. In the region between $\mathrm{C}$-mode and L-mode, the drain voltage reaches the knee region, which means that the amplifier's output power now depends on both input power and supply voltage. As the amplifier is operating close to compression, the efficiency in this mode is high. The fact that $P_{\text {out }}$ now depends on the supply voltage allows us to reduce $P_{\text {out }}$ without having to reduce the drive level. By reducing the $P_{\text {out }}$ of the peaking amplifier, the current it feeds into the common load is reduced, reducing the load-pulling effect on the main amplifier. This in turn leads to a higher load impedance than is usually presented to the main amplifier, and that is to prevent load-line interaction with the knee region. The main amplifier is thus being pushed further into compression further than before, leading to a decrease in gain and increase in distortion. This reduction in gain can however be used to shape the AM-AM characteristic of the DPA, e.g. to make it more linear or to shape it for easier linearisation. Operating the main amplifier in compression increases intermodulation distortion though, necessitating a trade-off.

Changing the peaking supply voltage $V_{\mathrm{D}, \mathrm{P}}$ does more than just shifting the peaking amplifier's load-line. As the value of the output capacitor $C_{\mathrm{DS}}$ of the peaking transistor depends on the drain voltage, a change of $V_{\mathrm{D}, \mathrm{P}}$ will change the shunt impedance of the peaking transistor. During the design of a DPA, an offset line is designed to transform the shunt impedance of the transistor to an open circuit at $Z_{\mathrm{p}, \text { off }}$, [9]. As the offset line can only transform one impedance to this highly resistive load, $Z_{\mathrm{p}, \text { off }}$ is only resistive at the design value $V_{\mathrm{D}, \mathrm{P}}=V_{\mathrm{D}, \mathrm{P} \text {,design. }}$. If the shunt impedance changes, the new impedance is no longer transformed to an open circuit by

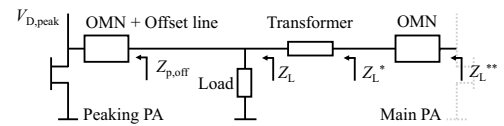

(a)

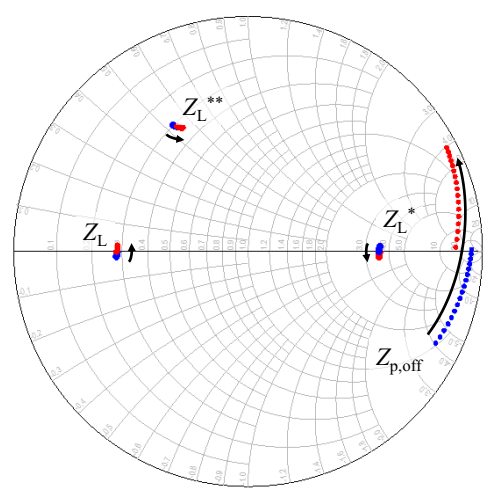

(b)

Fig. 3. Definition of reference planes (a), Impedances at different reference planes at a single frequency, arrows indicate increasing $V_{\mathrm{D}, \mathrm{P}}$; red and blue trajectories represent different values of $V_{\mathrm{D}, \mathrm{P}, \text { design }}(\mathrm{b})$

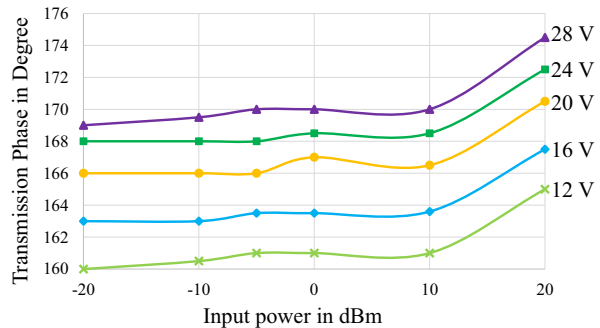

Fig. 4. Measured transmission phase of the main amplifier for a non-excited peaking amplifier in deep class $\mathrm{C}$ with different values for $V_{\mathrm{D}, \mathrm{P}}$ using the amplifier in Fig. 8.

output matching network (OMN) and offset line. This means that the impedance $Z_{\mathrm{p} \text {,off }}$ now has an imaginary component. As $V_{\mathrm{D}, \mathrm{P}}$ starts to deviate from $V_{\mathrm{D}, \mathrm{P} \text {,design }}, Z_{\mathrm{p} \text {,off }}$ takes on an increasingly large imaginary component, as shown in Fig. 3. If the design voltage $V_{\mathrm{D}, \mathrm{P} \text {,design }}$ is the maximum supply voltage value $V_{\mathrm{D}, \mathrm{P}}$ can assume, the imaginary component grows negatively for decreasing values of $V_{\mathrm{D}, \mathrm{P}}$ as this leads to a reduction in $C_{\mathrm{DS}}$. If $V_{\mathrm{D}, \mathrm{P}, \mathrm{design}}$ is the minimum value $V_{\mathrm{D}, \mathrm{P}}$ can assume, the imaginary components grows in positive direction for increasing values of $V_{\mathrm{D}, \mathrm{P}}$.

This change in load impedance results in a change of output phase as the measurement results in Fig. 4 show.

\section{Simulation}

For this simulation, an asymmetric DPA was designed utilising $10 \mathrm{~W}$ and $25 \mathrm{~W}$ Wolfspeed GaN HEMTs. All simulations are conducted at $2.4 \mathrm{GHz}$ to show their applicability for modern communication bands. The main amplifier is biased in class $\mathrm{B}$, the peaking amplifier in class $\mathrm{C}$; both gate voltages and the main amplifier's supply voltage are kept constant. Subjecting the DPA to an input power sweep for a number of different peaking supply voltages yields the results shown 

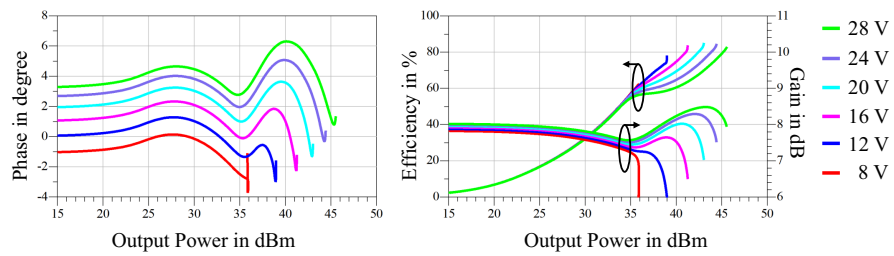

Fig. 5. Simulation result of the asymmetric DPA with class $\mathrm{C}$ peaking amplifier and compressed main amplifier from Fig. 1a with its peaking supply voltage swept from $8 \mathrm{~V}$ to $28 \mathrm{~V}$ in $4 \mathrm{~V}$ steps

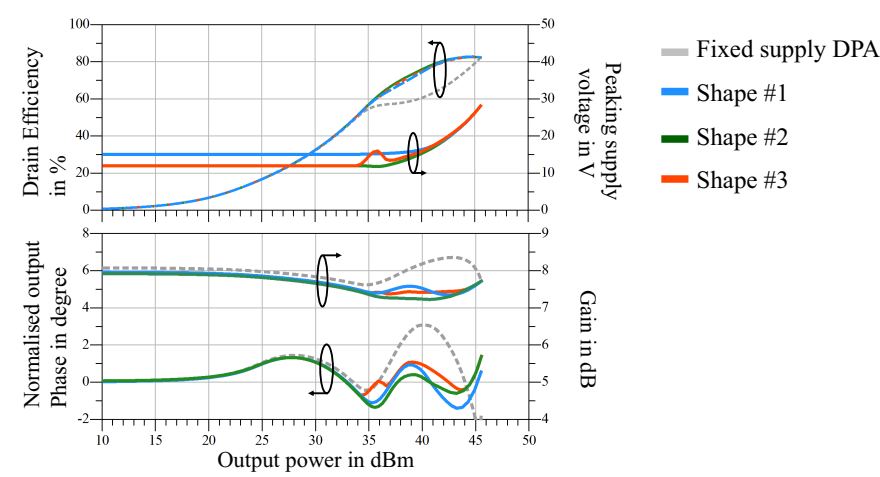

Fig. 6. Efficiency, AM/AM and AM/PM Distortion and the corresponding supply voltage shape for different shaping functions and a fixed supply voltage

in Fig. 5 which details the levels of freedom available in terms of efficiency, gain and phase variation. The supply voltage can only attain one value at a time, so distortion and efficiency will have to traded-off against each other by defining $V_{\mathrm{D}, \mathrm{P}}=f_{\mathrm{ET}}\left(v_{\text {in }}\right)$. As a result of $C_{\mathrm{DS}}$ depending on $V_{\mathrm{D}, \mathrm{P}}$, the difference between the phases at high $V_{\mathrm{D}, \mathrm{P}}$ at PEP and low $V_{\mathrm{D}, \mathrm{P}}$ at low drive power is lower than the difference between the phases at high $V_{\mathrm{D}, \mathrm{P}}$ at $\mathrm{PEP}$ and high $V_{\mathrm{D}, \mathrm{P}}$ at low drive powers as shown in Fig. 5. This indicates that as long as $V_{\mathrm{D}, \mathrm{P}}$ is low for low drive levels, and then increases, it will reduce the $\mathrm{AM} / \mathrm{PM}$ distortion. The shape the shaping function $f_{\mathrm{ET}}$ can attain is ultimately limited by the dynamic power supply (DPS) and the control circuitry. Analogue ET systems would typically limit the function to simple shapes such as linear or quadratic, see (1) where $v_{\text {in }}$ is normalised to its maximum value and $x$ is 2 for the linear and 4 for the quadratic function.

$$
V_{\mathrm{D}, \mathrm{P}}=\left(V_{\max }-V_{\min }\right) \cdot v_{\mathrm{in}}^{x}+V_{\min }
$$

Using a quadratic function and setting the minimum voltage $V_{\text {min }}$ to $15 \mathrm{~V}$ leads to the results called 'Shape \#1' in Fig. 6. This simple function leads to significant efficiency improvements while also reducing the gain and phase variation. To see how this relates to linearity, the DPA is subjected to a QAM16 signal. The results in Tab. I show that the quadratic function improves EVM and ACPR considerably.

Digital systems offer more freedom than analogue solutions, and allow almost arbitrary definitions of envelope functions. One obvious function to implement is the one that tracks the highest efficiency, called 'Shape \#2' in Fig. 6. This increases efficiency and AM/PM linearity, at the cost of an increased
TABLE I

EVM AND ACPR OF A $200 \mathrm{KHZ}$ 16-QAM SIGNAL FOR DIFFERENT SHAPING FUNCTIONS AT THE SAME PEP, 36 W, COMPARE FIG. 6

\begin{tabular}{c|c|c|c} 
& Shape & EVM in \% & Low/High ACPR $(\mathrm{dBc})$ \\
\hline Fixed Voltage & - & 3.6 & $-33.6 /-32.5$ \\
Quadratic ET Function & $\# 1$ & 1.45 & $-44.7 /-42.4$ \\
Maximum $\eta$ Function & $\# 2$ & 2.38 & $-39.0 /-37.6$ \\
4th order Function, PD & $\# 3$ & 1.86 & $-46.6 /-45.5$
\end{tabular}

AM/AM distortion. Reducing the peaking amplifier's supply voltage significantly not only increases its efficiency, it also drive the main amplifier further into compression, which increases its efficiency, while decreasing its gain. Having the ability to define the envelope shaping function almost arbitrarily allows the full access to the design space from Fig. 5. One possibility to use this freedom is to increase the linearisability of the amplifier. In this context, linearisability means the ease with which the amplifier can be pre-distorted. As the average base station size reduces with future mobile communication systems, the base-band part becomes an increasingly large chunk of base station total power consumption so sacrificing PA efficiency to make the amplifier easier to linearise may well increase the overall efficiency of the base station. Looking at the amplifier from the point of view of the pre-distorter, we want $\mathrm{AM} / \mathrm{AM}$ and $\mathrm{AM} / \mathrm{PM}$ distortions that are easily described mathematically. By manipulating the AM/AM characteristic to a shape that can achieves this, numerical complexity required by the DPD can be reduced. One way to achieve this is to construct a power series that approximates the AM/AM characteristic for low power behaviour, keeps within the allowed drain voltage limits and ends up at the maximum power. To keep the DPD complexity low, the number of coefficients needs to be kept as small as possible. Fig. 7 shows a 4th order function that fulfils the requirements, 'Shape \#3'. It also shows the connection between this function and the peaking amplifier's supply voltage. Using this connection, the shaping function $f_{\mathrm{ET}, 4}$, also can be created. Applying this shaping

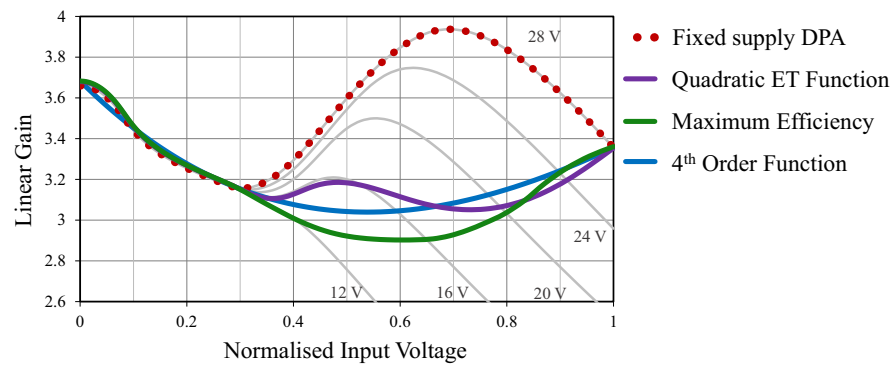

Fig. 7. Power series function compared to quadratic and maximum efficiency shaping functions from Fig. 6

function $f_{\mathrm{ET}, 4}$ to the peaking amplifier results in the gain shown in Fig. 7. By subjecting the input to the inverse function of $f_{\mathrm{g}, 4}$, the 'Shape \#3' profile and the results in Fig. 6 are achieved. These results show that efficiency is increased considerably and almost reaches the maximum efficiency function. The 
$\mathrm{AM} / \mathrm{AM}$ distortion is reduced considerably, as is the AM/PM distortion, just like with the other functions. These reductions in distortion directly translate into improved EVM and ACPR when the amplifier is subjected to a QAM16 signal, cf. Tab. I. Using a non-monotonic function will increase the bandwidth required by the DPS however, necessitating a trade-off.

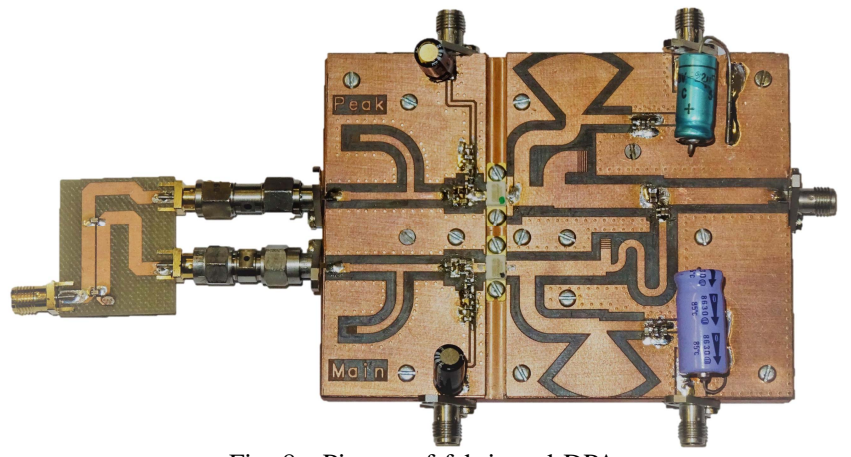

Fig. 8. Picture of fabricated DPA

\section{Measurements}

To verify the simulations, an amplifier was fabricated, as shown in Fig. 8, and subjected power sweeps for a range of peaking amplifier supply voltages. As expected, adjusting the peaking amplifier's supply voltage does increase the efficiency of the DPA in the high power region, see Fig. 9, albeit not as strongly as predicted in the simulation. Also, lowering $V_{\mathrm{D}, \mathrm{P}}$ below $22 \mathrm{~V}$ has much less impact on the efficiency than expected. The impact of the reduced $V_{\mathrm{D}, \mathrm{P}}$ on the gain in the low power region and phase is more distinctive than in the simulation. When changing $V_{\mathrm{D}, \mathrm{P}}$ from $28 \mathrm{~V}$ to $20 \mathrm{~V}$, the phase moves by $6^{\circ}$ in the measurement, significantly more than the $1.5^{\circ}$ suggested in simulation. This indicates that model and reality diverge when it comes to the bias dependence of $C_{\mathrm{DS}}$.

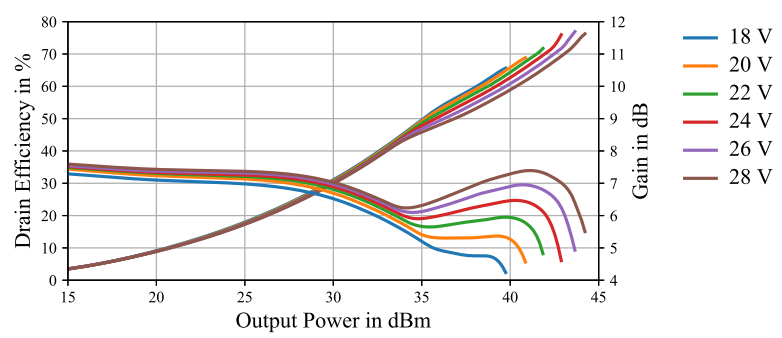

(a)

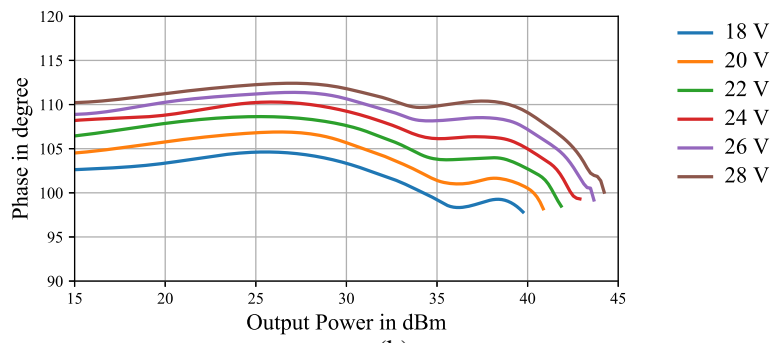

(b)

Fig. 9. Measurement results of the DPA in Fig. 8 for different $V_{\mathrm{D}, \mathrm{P}}$; drain efficiency and gain (a), transmission phase (b)

\section{OUTLOOK}

We have shown how applying supply voltage modulation to the peaking amplifier reduces AM/PM distortion and increases efficiency in a narrowband DPA. As the impact of the non-linear $C_{\mathrm{DS}}$ changes with frequency, the applicability of this approach in wideband applications is subject to further research. Measurements of the amplifier with a modulated supply voltage will be used to verify the influence of the shaping functions.

\section{CONCLUSION}

We proposed a combination of asymmetric DPA and ET in which the drain voltage of the peaking amplifier is modulated. This not only increases efficiency, but has also been shown to inherently improves the AM/PM distortion due to the nonlinear $C_{\mathrm{DS}}$. We showed that the demonstrated approach is able to achieve a high efficiency over a dynamic range of $11 \mathrm{~dB}$ with improved linearity. Even simple functions with no predistortion and a supply voltage dynamic range of only $5.4 \mathrm{~dB}$ result in high efficiencies. The possibility of configuring the tracking function to further increase efficiency or to shape the $\mathrm{AM} / \mathrm{AM}$ characteristic to greatly facilitate linearisability was demonstrated. We verified the simulations and showed in this case the DPS needs a dynamic range of $7.4 \mathrm{~dB}$ to achieve the high efficiency over the $11 \mathrm{~dB}$ of dynamic range.

\section{ACKNOWLEDGEMENT}

The authors would like to thank Wolfspeed for the donation of the transistors.

\section{REFERENCES}

[1] P. J. Tasker, V. Carrubba, P. Wright, J. Lees, J. Benedikt, and S Cripps, "Wideband pa design: The "continuous" mode of operation," in 2012 IEEE Compound Semiconductor Integrated Circuit Symposium (CSICS), Oct. 2012, pp. 1-4.

[2] P. Reynaert, "Polar modulation," IEEE Microwave Magazine, vol. 12, no. 1, pp. 46-51, Feb. 2011.

[3] J. Cha, Y. Yang, B. Shin, and B. Kim, "An adaptive bias controlled power amplifier with a load-modulated combining scheme for high efficiency and linearity," in Microwave Symposium Digest, 2003 IEEE MTT-S International, IEEE, vol. 1, 2003, pp. 81-84.

[4] B. Kim, J. Choi, D. Kang, and D. Kim, "Optimized envelope tracking operation of doherty power amplifier," Signals, Circuits and Systems (SCS), 2009 3rd International Conference on, pp. 1-5, 2009.

[5] Y. Cho, K. Moon, J. Kim, B. Park, and B. Kim, "Linear doherty power amplifier with adaptive bias circuit for average power-tracking," in 2016 IEEE MTT-S International Microwave Symposium (IMS), May 2016, pp. $1-3$.

[6] M. Thian and P. Gardner, "Envelope-tracking-based doherty power amplifier," International Journal of Electronics, vol. 97, no. 5, pp. 525530, 2010.

[7] S. Cripps, Advanced techniques in rf power amplifier design. Artech House, 2002.

[8] E. McCune, Dynamic power supply transmitters. Cambridge University Press, 2015, Cambridge Books Online.

[9] R. Quaglia, M. Pirola, and C. Ramella, "Offset lines in doherty power amplifiers: Analytical demonstration and design," IEEE Microwave and Wireless Components Letters, vol. 23, no. 2, pp. 93-95, Feb. 2013. 